\title{
Analysis of the Relationship between Burnout and Empathy Ability among Teachers in Special Education Schools
}

\author{
Xie Ma, Ting Deng ${ }^{*}$, Danna Luo, and Jinjing Ma \\ Department of Education, Yunnan Normal University, 650500, KunMing, China
}

\begin{abstract}
In order to explore whether the empathy ability of Chinese special education school teachers has an impact on their burnout, this study investigated 156 special education school teachers using the Empathy Competence Scale and the Burnout Scale. The results are as follows : (1)The scores of various dimensions of burnout of teachers in special education schools are all lower than the theoretical median, and the overall level of job burnout of this group is low. (2)Except imagination was not correlated with emotional exhaustion, other dimensions of empathy ability were significantly correlated with all dimensions of burnout (all $p<0.05$ ). (3)Regression analysis found that perspective taking could negatively predict emotional exhaustion and low personal accomplishment (all $p$ $<0.05$ ). Empathy concern could negatively predict low personal achievement $(p<0.05)$. Personal distress positively predicted emotional exhaustion, depersonalization, and low personal accomplishment (all $p$ $<0.001)$. Imagination could negatively predict emotional exhaustion and depersonalization (all $p<0.05$ ).
\end{abstract}

\section{Introduction}

According to the 2019 China Education Statistics Yearbook, there are currently 2,192 special education schools in China, with 62,400 teachers and 794,600 students. Compared with 2010, the number of special education schools increased by 486, the number of special education school teachers increased by 22,700 , with growth rates of $28.49 \%$ and $57.18 \%$ respectively, whereas the number of special education students in school increased by 369,000 , with a growth rate of $86.70 \%$. Therefore, the number of special education schools and the growth of teachers can hardly keep up with the demand for special education students, and there is an obvious shortage of teachers in special education schools. At the same time, some researchers point out that teachers in special education schools, as a highrisk group, are prone to burnout symptoms such as low job satisfaction, low self-efficacy, and emotional exhaustion, which may lead to their resignation or application for other

\footnotetext{
* Corresponding author: Antinar@aliyun.com

Xie Ma \& Ting Deng: These authors contributed equally to this work and should be considered cofirst authors
} 
positions in school, resulting in a higher staff turnover rate, which is not conducive to the development of special education [1-3]. Therefore, burnout, professional identification, professional efficacy, and other related problems of teachers in special education schools have attracted more and more researchers' attention [4-5].

Previous studies have shown that empathy ability can have a significant impact on an individual's career development. N.Li[6]et al. (2016) found that total empathy ability scores, and perspective taking were negatively related to emotional exhaustion, and perspective taking was negatively related to depersonalisation, and positively related to personal accomplishment .T.Wang[7]et al. (2015) also found that the higher the scores of imagination and empathy care, the less likely burnout is to occur, and the increase of personal distress will enhance burnout. At present, studies on the relationship between burnout and empathy ability are mostly focused on the medical and nursing communities, and lack of studies on teachers in special education schools. Teachers in special education schools and medical groups are also in a complex working environment, and they have to face special children with physical and mental disabilities for a long time. Some researchers believe that the particularity of the objects faced will bring teachers unique psychological experience, thus increasing work pressure and leading to emotional fatigue [8-9]. Therefore, teachers in special education schools need to be highly empathy ability to face students with hearing disabilities, autism, cerebral palsy and other disabilities, and to promote better professional development. This study focuses on the phenomenon of burnout among teachers in special education schools, takes empathy ability as the starting point, investigates the current situation of burnout, and analyzes the relationship between burnout and empathy ability in order to provide a reference for the professional development and research of teachers in special education schools.

\section{Methods}

\subsection{Subjects}

A total of 156 valid questionnaires were collected from teachers of special education schools in different provinces and cities. There were 37 male teachers (23.72\%) and 119 female teachers (76.28\%); 14 (8.97\%) had a specialist degree or below, 131 (83.98\%) had a bachelor's degree and 11 (7.05\%) had a postgraduate degree or above; 53 (33.97\%) were senior teachers or above, 49 (31.41\%) were first-grade teachers and 54 (34.62\%) were second-grade teachers and below. Age ranged from 20 to 58 years old, with an average age of 36.67 years; teaching experience ranged from 2 months to 37 years, with an average of 13.86 years; the average number of lessons was 13.76 .

\subsection{Measures}

Burnout Scale: A 16-question generalized burnout scale compiled by Maslach, divided into 3 dimensions: emotional exhaustion (positive scoring), depersonalization (positive scoring), and low personal accomplishment (reverse scoring). A 5-point scale was used, ranging from 1 (disagrees) to 5 (agrees). Internal consistency tests found the questionnaire to had good reliability and validity, with a total scale reliability of 0.890 and homogeneous reliability for the dimensions of emotional exhaustion, depersonalisation and low personal accomplishment of 0.919, 0.757 and 0.846 respectively.

Empathy Ability Scale: Interpersonal Response Indicator Scale was developed by Zhan's, is a 22-question scale divided into four dimensions: perspective taking, empathy concern, imagination, and personal distress. A 5-point scale was used, ranging from 1 
(disagrees) to 5 (agrees), with higher scores indicating greater empathy ability. The questionnaire has a good fit index and the internal consistency test found a reliability of 0.723, with good internal consistency reliability.

\subsection{Analysis}

Statistical analyses were performed using SPSS software, version 22.

\section{Results}

\subsection{Burnout status}

As shown in Table 1, the scores of emotional exhaustion, depersonalization and low personal accomplishment of teachers in special education schools are respectively 12.37 , 10.49 and 12.13, all of which are lower than the theoretical median, indicating that the emotional exhaustion and depersonalization of teachers in special education schools are not serious and they have a strong personal accomplishment.

Table 1. The overall state of burnout $(n=156)$

\begin{tabular}{|c|c|c|c|}
\hline & Emotional exhaustion & Depersonalisation & Low personal accomplishment \\
\hline $\mathrm{M}$ & 12.37 & 10.49 & 23.87 \\
\hline SD & 4.67 & 3.82 & 4.30 \\
\hline
\end{tabular}

Note: ${ }^{*} p<0.05,{ }^{* *} p<0.01,{ }^{* * *} p<0.001$

\subsection{Analysis of the correlation between burnout and empathy ability}

The results of correlation analysis (see Table 2) showed that Perspective taking was negatively correlated with emotional exhaustion, depersonalization, and low personal accomplishment (all $p<0.05$ ). Empathy concern was negatively correlated with emotional exhaustion, depersonalization, and low personal accomplishment (all $p<0.01$ ). There was a significant positive correlation between personal distress and emotional exhaustion, depersonalization, and low personal accomplishment (all $p<0.01$ ). Imagination was not associated with emotional exhaustion, but was negatively associated with depersonalization and low personal accomplishment (all $p<0.05$ ).

Table 2. The correlation coefficient between burnout and empathy ability ( $n=156)$

\begin{tabular}{|c|c|c|c|c|}
\hline & $\begin{array}{c}\text { Perspective } \\
\text { taking }\end{array}$ & $\begin{array}{c}\text { Empathy } \\
\text { concern }\end{array}$ & $\begin{array}{c}\text { Personal } \\
\text { distress }\end{array}$ & Imagination \\
\hline Emotional exhaustion & $-0.253^{* *}$ & $-0.074^{* *}$ & $0.389^{* *}$ & -0.130 \\
\hline Depersonalisation & $-0.180^{*}$ & $-0.211^{* *}$ & $0.354^{* *}$ & $-0.237^{* *}$ \\
\hline $\begin{array}{c}\text { Personal } \\
\text { accomplishment }\end{array}$ & $0.357^{* *}$ & $0.277^{* *}$ & $-0.275^{* *}$ & $0.170^{*}$ \\
\hline
\end{tabular}

Note: ${ }^{*} p<0.05,{ }^{* *} p<0.01, * * * p<0.001$ 


\subsection{Regression analysis of burnout and empathy ability}

To explore the predictive effect of empathy ability on burnout among special education school teachers, regression analyses were conducted using the dimensions of empathy ability as predictive variables and the dimensions of burnout as outcome variables for special education school teachers (see Table 3). It was found that perspective taking into the regression equation could negatively predict emotional exhaustion $(p<0.05)$ and low personal accomplishment $(p<0.001)$. Empathy concern into the regression equation could negatively predict low personal accomplishment $(p<0.05)$; Personal distress entered the regression equation and positively predicted emotional exhaustion $(p<0.001)$, depersonalization $(p<0.001)$, and low personal accomplishment $(p<0.001)$; Imagination entering the regression equation negatively predicted emotional exhaustion $(p<0.05)$ and depersonalization $(p<0.01)$.

Table 3. Regression analysis of burnout and empathy ability ( $n=156)$

\begin{tabular}{|c|c|c|c|c|c|}
\hline & & $\boldsymbol{\beta}$ & $\boldsymbol{t}$ & $\boldsymbol{R}^{2}$ & $\boldsymbol{F}$ \\
\hline Emotional exhaustion & Perspective taking & -0.199 & $-2.535^{*}$ & 0.230 & $11.301^{* * *}$ \\
\hline & Empathy concern & 0.046 & 0.589 & & \\
\hline & Personal distress & 0.406 & $5.594^{* * *}$ & & \\
\hline & Imagination & -0.116 & -1.466 & & \\
\hline Depersonalisation & Perspective taking & -0.062 & -0.793 & 0.230 & $11.306^{* * *}$ \\
\hline & Empathy concern & -0.106 & -1.362 & & \\
\hline & Personal distress & 0.391 & $5.387^{* * *}$ & & \\
\hline Low personal accomplishment & Perspective taking & 0.280 & $3.580^{* * *}$ & 0.233 & $11.480^{* * *}$ \\
\hline & Empathy concern & 0.153 & $1.977^{*}$ & & \\
\hline & Personal distress & -0.283 & $-3.909^{* * *}$ & & \\
\hline & Imagination & 0.075 & 0.955 & & \\
\hline
\end{tabular}

Note: ${ }^{*} p<0.05,{ }^{* *} p<0.01,{ }^{* * *} p<0.001$

\section{Discussions}

There are different opinions about the level of burnout of teachers in special education schools. For example, P.Maria [10]et al.(2008)used Greek special education school teachers as subjects and found that this group did not show significant burnout, and D.W.Emery [11](2010) noted that special education school teachers in the United States showed low job satisfaction, low self-efficacy, high levels of stress and burnout, and a high attrition rate. The results of this study showed that the phenomenon of burnout of teachers in special education schools is not serious and show high levels of personal accomplishment, which is consistent with the findings of P.Maria [10]et al. This may be related to the pressure of admission to school, where children in special education schools focus on developing basic 
skills such as self-care and emotional control, and teachers face less pressure of admission to school. In addition, in relation to salary levels and school management, teachers in special education schools may be more enthusiastic about their work because they receive an additional monthly allowance and are relatively independent in their professional decision-making and less restricted by others.

An analysis of the relationship between burnout and empathy ability in special education teachers found that all dimensions of empathy ability were significantly related to the other dimensions of burnout, except the imagination was not significantly related to personal accomplishment, indicating that empathy ability was an important factor influencing their burnout, which is consistent with the findings of C. Williams et al. [12-13] In this study, we conducted a regression analysis of the relationship between the two. We found that the perspective taking of empathy ability, empathy concern, personal distress, and imagination were all included in the regression equation. The perspective taking of empathy ability could negatively predict emotional exhaustion and low personal achievement. Empathy concern can negatively predict low personal accomplishment. Personal distress positively predicted emotional exhaustion, depersonalization and low personal accomplishment. Imagination can negatively predict depersonalization. Therefore, it can be concluded that training the empathy ability of teachers in special education schools can be one of the effective methods to prevent their burnout.

As one of the basic abilities of special education school teachers, the correct understanding and rational use of empathy ability will help them better understand the real feelings and special needs of special children in teaching practice and make reasonable responses, which can effectively alleviate burnout [14]. In order to improve the empathy ability of teachers in special education schools, the following suggestions are put forward: Firstly, reduce personal distress. Studies have found that support from society, school and family can relieve teachers' personal distress and work pressure, and thus reduce burnout. Secondly, enhance the choice of perspective taking and imagination. Perception taking and imagination belong to cognitive empathy. The theoretical model of lifelong development of empathy points out that cognitive empathy is postnatal acquired and improves with age. Therefore, it is suggested to enhance their empathy ability through cognitive empathy training so as to effectively reduce burnout. Finally, establish a sense of boundary. It is necessary to establish the boundary of one's own ability and understand the limited ability of oneself, but also to establish the boundary between work and life, so as not to let the negative emotional events encountered during work and the personal distress of students affect personal life.

Supported by: National Natural Science Foundation of China (No. 31660282) and Project of philosophy and social science planning in Yunnan Province (QN2017043)

\section{References}

1. J. Eichinger,Int J Disabil Dev Educ, Job stress and satisfaction among special education teachers: effects of gender and social role orientation, 47, 397-412(2000)

2. A. Roach, Ph. D, Teacher burnout: special education versus regular education, (2009)

3. R. Küüksüleymanoglu, Int J Spec Educa, Burnout syndrome levels of teachers in special education schools in Turkey, 26, 53-63 (2011)

4. B. Gulsan, Education and Science, A comparison of female and male school administrators' burnout levels controlling for perceived social support, 38, 3-18 (2013)

5. B. Filiz, Soc Behav Personal, Examining the burnout of academics in relation to job satisfaction and other factors, 34, 1151-1160 (2006) 
6. N. Li, C. Tong, M. Zhang, et al, Journal of Nursing Science, Correlation between empathy ability and job burnout among emergency nurses, 31, 61-62 (2016)

7. T. Wang, W. L. Wang, J. F. Hong, et al, Chinese Nursing Management, Subjective well-being and job burnout of clinical nurses: the role of empathy, 15, 280-284 (2015)

8. R. Eugene Hughes, Int J Hum Resour Man, Deciding to leave but staying: teacher burnout, precursors and turnover, 12, 288-298 (2001)

9. C. M. Kokkinos, Stress Health, Factor structure and psychometric properties of the Maslach Burnout Inventory-Educators Survey among elementary and secondary school teachers in Cyprus, 22, 25-33 (2006)

10. P. Maria, A. Ioannis, Int J Disabil Dev Educ, Burnout, Job Satisfaction and Instructional Assignment-related Sources of Stress in Greek Special Education Teachers, 55, 61-76 (2008)

11. D. W. Emery, B. Vandenberg, Int J Spe Educ, Special education teacher burnout and act, 25, 119-131 (2010)

12. C. Williams, Res Nurs Health, Empathy and burnout in male and female helping professionals, 12, 169-178 (2010)

13. O. Yuguero, C. Forné, M. Esquerda, et al, Medicine, Empathy and burnout of emergency professionals of a health region, 96, e8030 (2017)

14. J. J. Lan, X. L. Guo, Z. L. Jia, et al, Chinese Journal of Special Education, The Effect of homebound instruction teachers' empathy ability on their attitude: the mediating role of professional identity, 58-63 (2020) 